\title{
Safety profile of chimeric antigen receptor T-cell immunotherapies (CAR-T) in clinical practice
}

\author{
Giulia Bonaldo ${ }^{1} \cdot$ Nicola Montanaro ${ }^{2} \cdot$ AlbertoVaccheri $^{1} \cdot$ Domenico Motola $^{1} \mathbb{C}$ \\ Received: 6 October 2020 / Accepted: 3 February 2021 / Published online: 20 February 2021 \\ (C) The Author(s) 2021
}

\begin{abstract}
Purpose Two chimeric antigen receptor T-cell (CAR-T) therapies have been approved in the United States (USA) in 2017 and Europe (EU) in 2018: axicabtagene ciloleucel and tisagenlecleucel. They contain the patient's own T cells, which are extracted, genetically modified, and reinfused. Alongside the good efficacy results, the assessment of safety profile of these new therapies represents a great challenge. Our aim was to analyze the reports of the adverse drug reactions (ADR) after CAR-T administration as occurred in the real clinical setting.

Methods We performed a retrospective observational study, collecting all the reports in EU (EudraVigilance, EV) and US (FAERS) databases of ADRs regarding axicabtagene ciloleucel and tisagenlecleucel. Both descriptive and statistical analyses were performed, the latter by using Reporting Odds Ratio (ROR).

Results A total number of 1426 reports of suspected ADRs were retrieved in EudraVigilance and FAERS. Patients' reported age reflected the age range for which the drugs are approved (18-64 years for axicabtagene ciloleucel and patients aged under 25 years for tisagenlecleucel). The most reported event was cytokine release syndrome (CRS), 185 events for tisagenlecleucel and 462 for axicabtagene ciloleucel in FAERS and 137 and 498, respectively, in EudraVigilance. A disproportionality was found comparing axicabtagene ciloleucel with tisagenlecleucel for the above-mentioned event: EV ROR 2.47, 95\% CI 2.22-2.74, FAERS 1.89, 1.70-2.10.

Conclusion CRS represents the major problem with the administration of CAR-T therapies. Our analysis has not revealed new ADRs; however, it supports the safety profile of CAR-T with new data from real clinical setting.
\end{abstract}

Keywords CAR-T $\cdot$ Safety profile $\cdot$ Clinical practice $\cdot$ ADR

\section{Introduction}

In the last years, personalized medicine in immuno-oncology reached great goals, and, among these, the most important one was the advent of CAR-T therapies. The acronym CAR-T stands for chimeric antigen receptor T-cell therapy, which consists of patient's genetically modified white blood cells yielded capable to perform antitumor activity using a lentiviral vector encoding an anti-CD19 chimeric antigen receptor-CAR

Domenico Motola

domenico.motola@unibo.it

1 Unit of Pharmacology, Department of Medical and Surgical Sciences, Alma Mater Studiorum University of Bologna, via Irnerio 48, 40126 Bologna, Italy

2 Pharmacology at the Alma Mater Studiorum, University of Bologna, Bologna, Italy (axicabtagene ciloleucel) or by retroviral transduction to express a chimeric antigen receptor (tisagenlecleucel). After lymphodepletion, the modified $\mathrm{T}$ cells reinfused in the patients can attach a specific antigen on the tumor cells. Two CAR-T therapies have been approved in Europe (August 2018), i.e., axicabtagene ciloleucel (Yescarta $\left.{ }^{\circledR}\right)[1,2]$ and tisagenlecleucel (Kymriah®) [3]. These therapies have been also approved in 2017 by the US Food and Drug Administration (US FDA) $[4,5]$.

Axicabtagene ciloleucel is approved for two types of blood cancer: diffuse large B-cell lymphoma (DLBCL) and primary mediastinal large B-cell lymphoma (PMBCL) [6]. Tisagenlecleucel is approved for the treatment of B-cell acute lymphoblastic leukemia (ALL) refractory or in relapse (posttransplant, second or later relapse) in children and young adults up to 25 years and for adult patients with relapsed or refractory DLBCL after two or more lines of systemic therapy [7]. Two main studies were performed with tisagenlecleucel in 
B-cell ALL (92 children and young adults) [8] and in DLBCL (165 patients) [9]. Twelve months after treatment, the survival probability was $70 \%$ and $40.2 \%$, respectively [10]. A main study in 111 patients with DLBCL and PMBCL was carried out for axicabtagene ciloleucel [11], showing a complete response in $47 \%$ of the patients and a partial response in the $19 \%$ [12]. Apart from the great efficacy results, the knowledge of the safety profile of these new therapies represents a great challenge. Both drugs must be administered in a qualified center for hematological malignancies with healthcare professionals trained in the management of possible adverse events. Patients undergo pre-medication, clinical assessment prior to infusion, and monitoring after infusion to minimize potential adverse events. Our aim was to evaluate all the adverse events reported following the administration of axicabtagene ciloleucel and tisagenlecleucel in the real clinical setting in Europe and in the USA.

\section{Methods}

Data were retrieved from the European database of suspected adverse drug reaction reports (EudraVigilance) using the online interface adrreports.eu [13] and from the US Food and Drug Administration Adverse Event Reporting system (FAERS) using a freely available and of public consultation dashboard. EudraVigilance is the system for the management and collection of suspected adverse events reported for medicines. It is maintained by the European Medicines Agency (EMA) on behalf of the European Union (EU). In November 2017, the new EudraVigilance system with enhanced functionalities was launched with an improvement of the collection of the individual case safety reports (ICSRs) from regulatory authorities and marketing authorization holders (MAHs) and of the detection of possible safety signals [14]. The FDA Adverse Event Reporting System (FAERS) is the database for the collection of all the reports of suspected ADRs submitted to FDA. All the reports are in a structured form that includes administrative information, patient's characteristics, suspected and concomitant drugs, and the adverse drug reactions. For our aim, we focused on the analysis of both antiCD19 chimeric antigen receptor T-cell immunotherapies (CAR-T). We retrieved all the reports with axicabtagene ciloleucel or tisagenlecleucel reported as suspected drugs. Considering that both drugs received a marketing authorization valid throughout the European Union on 23 August 2018, we considered the period between September 2018 and 22 October 2019 for our analysis in EudraVigilance. For FAERS analysis, we considered the period between September 2017 (first CAR-T approval date) and 30 June 2019 (date of the most recent update of the dashboard).

\section{Descriptive analysis}

The extracted reports were identified by a unique EU Local Number in EudraVigilance or by a unique Case ID in FAERS. The information reported was the report type (spontaneous or from clinical studies), receipt date, primary source qualification (healthcare professional or non-healthcare professional), patient age group, patient sex, preferred term MedDRA, seriousness criteria, and suspect and concomitant drugs. The MedDRA is a standardized medical terminology that allows to report adverse events in the same way all over the world. It is used by both databases, and this allows an easy comparison of the data reported [15]. This dictionary has a hierarchic terminology: several preferred terms (PTs) are grouped in one System Organ Class (SOC) by etiology, manifestation site, or purpose.

We analyzed all the reports related to axicabtagene ciloleucel or tisagenlecleucel. A descriptive analysis was performed to evaluate all the reported adverse events. It should be noted that each report could contain one or more adverse events. For each drug, it was checked if the reported events were listed in the corresponding Summary of the Product Characteristics (SPCs) to ascertain the notoriety of the adverse reactions.

\section{Statistical analysis}

A case-non-case analysis was performed by using the Reporting Odds Ratio (ROR) with 95\% confidence interval as statistical parameter. ROR allows a quantitative approach by the use of contingency tables. The aim is to compare the frequency of a drug-reaction pair with all the others in the database or with different therapeutic regimens. If ROR is $>1$, an increased frequency for the drug-reaction pair can be assumed. Considering the specificity of the treatments analyzed, the use of the whole database was considered not appropriate. Therefore, a disproportionality analysis was carried out by comparing the two treatments. Other articles in literature have used a similar approach when comparing different therapeutic regimens [16]. The proportions of the adverse drug reactions to axicabtagene ciloleucel were compared to those with tisagenlecleucel. The denominator was the total number of adverse drug reactions reported for both drugs.

\section{Results}

Table 1 shows the most reported adverse events for both CAR-T therapies in EudraVigilance and FAERS. For each event, it is also indicated whether the ADR is reported in the Summary of Product Characteristic of the corresponding CAR-T therapy (pointed out with *); it is not directly reported 


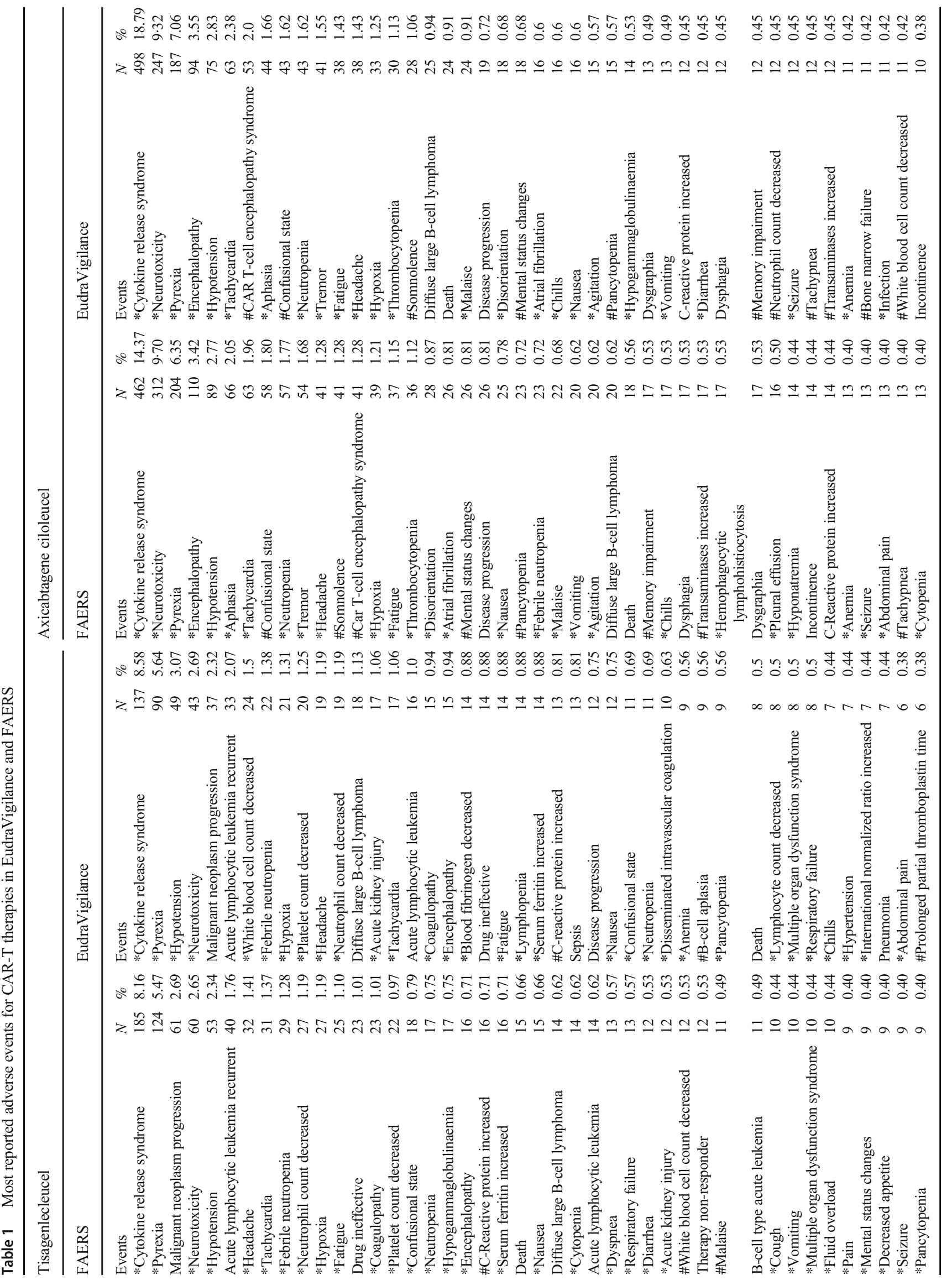


in the SPC but linked to other reported events (\#), or it is not reported at all.

\section{Descriptive analysis of axicabtagene ciloleucel}

\section{EudraVigilance}

We retrieved 683 reports of suspected adverse reactions referred to axicabtagene ciloleucel. One hundred ninety-five reports were related to female patients $(28.6 \%)$ and 306 $(44.8 \%)$ to males. For 182 reports $(26.6 \%)$, sex of the patients was not reported. Table 2 shows the reports classified by age and sex in the two databases. Most of the patients (277; $40.6 \%$ ) belonged to the $18-64$ years age class. Only one report concerned a female patient of the age class $12-17$ years and 2 patients, one male and one female, aged $>85$ years. The great majority of the ADRs $(673,98.5 \%)$ were reported by healthcare professionals. Overall, 2650 adverse events were reported for axicabtagene ciloleucel. The top five most reported were cytokine release syndrome (498 events, 18.8\%), neurotoxicity $(247,9.3 \%)$, pyrexia $(187,7.1 \%)$, encephalopathy $(94,3.6 \%)$, and hypotension $(75,2.8 \%)$. The most reported concomitant therapies were acyclovir, levetiracetam, and ondansetron and, to a less extent, tocilizumab.

\section{FAERS}

We retrieved 743 reports of suspected adverse reactions referred to axicabtagene ciloleucel in FAERS. Overall, one report $(0.1 \%)$ was reported in 2017, $440(59.2 \%)$ in 2018, and 302 (40.7\%) in 2019 until the 30th of June. Report for male patients were $387(52.1 \%)$ and those for females were 226 (30.4\%). Sex was not stated in 130 reports $(17.5 \%)$. The great majority of the reports were related to $18-64$ years age class $(45.4 \%)$. Only two reports concerned female patients aged $>85$ years. Table 2 shows reports classified by age and sex in the two databases. The vast majority of reporters were healthcare professionals $(699,94.08 \%)$, whereas 18 reports $(2.42 \%)$ came from consumers. Overall, 3215 events were reported for axicabtagene ciloleucel in FAERS. The top five most reported events were cytokine release syndrome (462 events, $62.2 \%$ ), neurotoxicity $(312,42.0 \%)$, pyrexia $(204,27.5 \%)$, encephalopathy $(110$, $14.8 \%)$, and hypotension $(89,11.98 \%)$. The most reported concomitant therapies were acyclovir, levetiracetam, allopurinol, fluconazole, fludarabine, cefepime, vancomycin, cyclophosphamide, ondansetron, and tocilizumab.

\section{Descriptive analysis of tisagenlecleucel}

\section{EudraVigilance}

In the same period, we retrieved 284 reports of suspected adverse reactions referred to tisagenlecleucel of which 137 


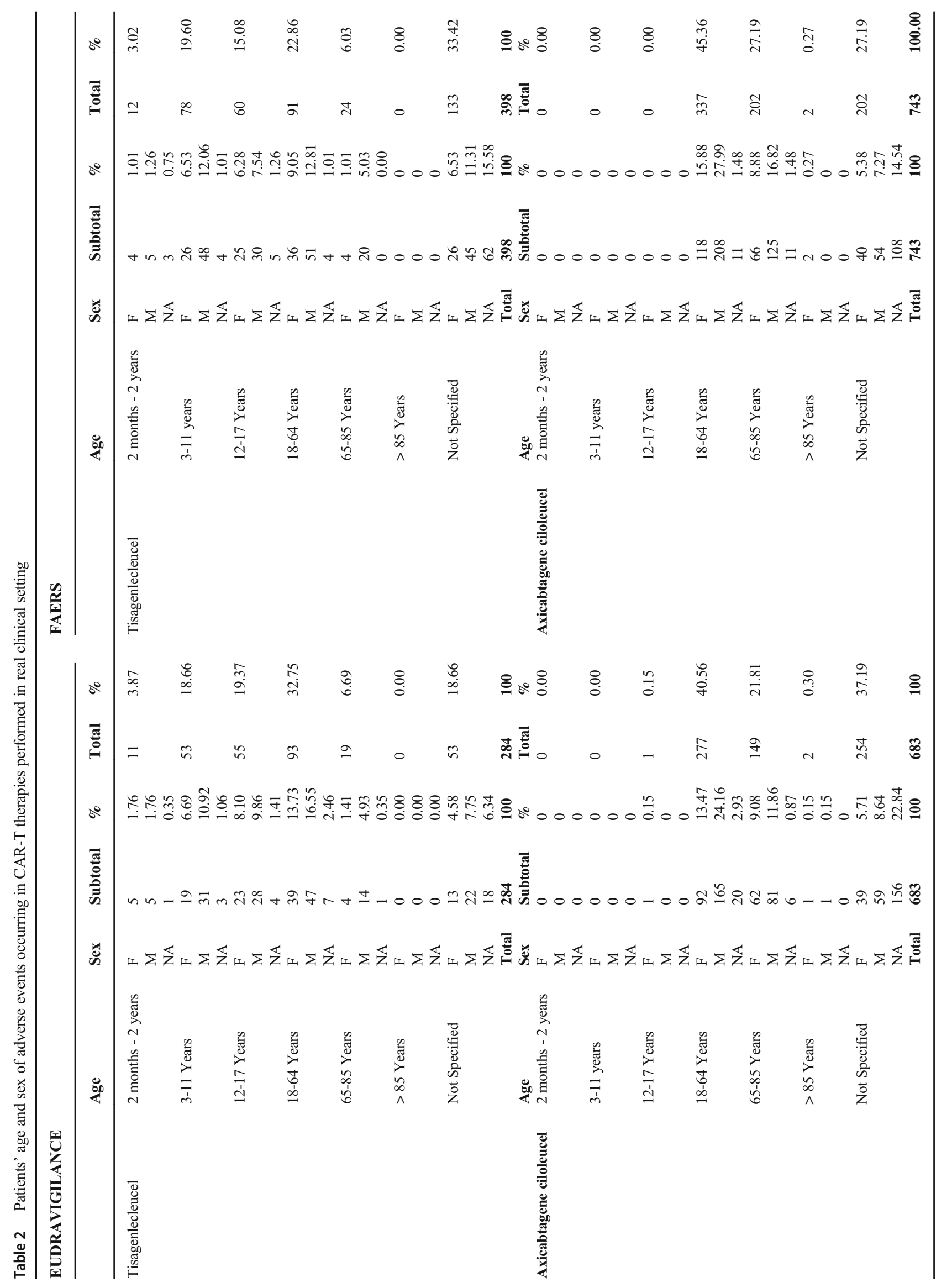


in 2018 and 147 in 2019. One hundred and three (36.3\%) were related to female patients and $147(51.8 \%)$ to males. For 34 reports $(12.0 \%)$, sex of the patients was not stated. All data are shown in Table 2. Overall, 119 reports regarded patients aged less than 18 years. In particular, 11 patients $(3.9 \%)$ were aged 2 months-2 years, $54(18.7 \%) 3-11$ years, and $55(19.4 \%)$ $12-17$ years. Ninety-three reports $(32.7 \%)$ referred to the age class $18-64$ years. The large majority $(96.1 \%)$ of the reports came from healthcare professionals. Overall, 1597 events were reported for tisagenlecleucel. The top five most reported events were cytokine release syndrome (137 events, $8.6 \%)$, pyrexia $(90,5.6 \%)$, hypotension $(49,3.1 \%)$, neurotoxicity $(43,2.7 \%)$, and malignant neoplasm progression ( 37 , $2.3 \%$ ). The most reported concomitant therapies were cyclophosphamide, fludarabine, levetiracetam, acyclovir, amlodipine, ondansetron, and tocilizumab.

\section{FAERS}

Overall, 398 reports were retrieved for tisagenlecleucel in FAERS. Of these, $10(2.5 \%)$ were reported in 2017, 179 $(45.0 \%)$ in 2018, and $209(52.5 \%)$ in 2019 until June 30. The half of the reports $(199,50.0 \%)$ concerned male patients, $121(30.4 \%)$ females, and for $78(19.6 \%)$, sex was not reported. The majority of the reports were related to minors, as shown in Table 2. Of the 91 patients in the age class 18-64 years, $54(59.3 \%)$ were patients $\leq 25$ years old. For 133 $(33.4 \%)$ patients, age class was not specified. The majority of reports $(79.4 \%)$ came from healthcare professionals, $20.4 \%$ from consumers. The overall events reported for tisagenlecleucel in FAERS were 2268. The top five most reported ones were cytokine release syndrome (185, 46.5\%), pyrexia $(124,31.2 \%)$, malignant neoplasm progression $(61$, $15.3 \%)$, neurotoxicity $(60,15.1 \%)$, and hypotension $(53$, $13.3 \%)$. The most reported concomitant medications were levetiracetam, acyclovir, allopurinol, fludarabine, voriconazole, cyclophosphamide, cefepime, cotrimoxazol, ondansetron, levofloxacin, and tocilizumab.

\section{Statistical analysis}

We compared the ADRs reported for axicabtagene ciloleucel with those for tisagenlecleucel in either database. Overall, 196 events were reported for both drugs in EudraVigilance and 243 in FAERS. A disproportionality was found for axicabtagene ciloleucel as compared to tisagenlecleucel concerning the following three events. The ROR values were cytokine release syndrome (EV ROR 2.47 [95\% CI 2.222.74], FAERS ROR 1.89 [1.70-2.10]), neurotoxicity (EV ROR, 3.71 [3.13-4.41], FAERS ROR 3.96 [3.42-4.57]), and pyrexia (EV ROR 1.27 [1.08-1.50], FAERS ROR 1.17 [1.00-1.37]). All these three events are acknowledged on the Summary of Product Characteristics (SPC) of the drugs. As shown in Table 3, among the drug-reaction pairs with higher and statistically significant ROR in both databases, we found aphasia (EV ROR 8.97 [3.47-23.22], FAERS ROR 15.82 [6.46-38.77]), CAR-T-cell-related encephalopathy syndrome (EV ROR 8.13[3.80-17.40], FAERS ROR 9.75 [3.7325.49]), atrial fibrillation (EV ROR 4.84 [1.11-21.11], FAERS ROR 9.24, [95\% CI 2.36-36.20]), and thrombocytopenia (EV ROR 4.56 [95\% CI 1.95-10.67], FAERS ROR 5.13 [95\% CI 2.49-10.53]).

\section{Discussion}

To the best of our knowledge, this is the first study aimed at evaluating and comparing the safety profile of the CAR-T therapies with the use of data of real clinical practice. Postapproval pharmacovigilance is considered crucial for the evaluation of CAR-T safety profile [17], as it allows long-term follow-up in a large and uncontrolled population. From the present analysis, no new and unexpected ADRs emerged in daily clinical practice, and this is reassuring. The patients experiencing an ADR with either drug were different in term of age. Most of axicabtagene ciloleucel patients were aged 18-64 years or more, whereas those receiving tisagenlecleucel were under 25 years of age and mainly minors. This reflects the indications for which the drugs have been approved: tisagenlecleucel is the only CAR-T therapy specifically approved for the treatment of patients $\leq 25$ years of age with $R /$ R B-cell acute lymphoblastic leukemia.

Proportion of male patients was higher with both drugs and databases. This does not point out a greater predisposition of male patients to develop an ADR, but it is rather related to the prevalence of the disease to be treated. Both diffuse large Bcell lymphoma (DLBCL) and acute lymphocytic leukemia (ALL) have a slightly higher incidence in males than females. The US National Cancer Institute reported that the number of new cases per 100,000 persons in the period 2012-2016 were 6.7 in males and 4.6 in female for DLBCL and 1.9 and 1.5, respectively, in female for ALL [18, 19].

A lower presence of consumers as reporters has been identified in EudraVigilance in comparison with FAERS, this confirming the lower tendency of patients to report ADRs in Europe [20]. Cytokine release syndrome (CRS) was the most reported ADR in both databases and for both drugs.

The pathophysiology of the syndrome is unclear, and recent studies showed that IL-6, IL-1, and nitric oxide produced by macrophages are involved in its course [21]. The vast majority of the others events reported in our study could be symptoms of this syndrome, which is characterized mainly by pyrexia and hypotension, the most reported events retrieved after CRS. Other ADRs associable with CRS are hypoxia, chills, cardiac adverse events (e.g., tachycardia, atrial fibrillation, and cardiac arrest), acute kidney injury, and also 
Table 3 Adverse events reported with axicabtagene compared to tisagenlecleucel in EudraVigilance and FAERS and Reporting Odds Ratio (ROR)

EUDRAVIGILANCE

\begin{tabular}{|c|c|c|c|c|c|}
\hline Events & $\mathrm{N}$ axicabtagene & $\mathrm{N}$ tisagenleucleucel & ROR & CI_low & CI_up \\
\hline Aphasia & 44 & 3 & 8.97 & 3.47 & 23.22 \\
\hline Tremor & 41 & 3 & 8.35 & 3.19 & 21.84 \\
\hline CART-cell-related encephalopathy syndrome & 53 & 4 & 8.13 & 3.80 & 17.40 \\
\hline Atrialfibrillation & 16 & 2 & 4.84 & 1.11 & 21.11 \\
\hline Thrombocytopenia & 30 & 4 & 4.56 & 1.95 & 10.67 \\
\hline Encephalopathy & 94 & 15 & 3.88 & 2.78 & 5.42 \\
\hline Neurotoxicity & 247 & 43 & 3.71 & 3.13 & 4.41 \\
\hline Somnolence & 28 & 5 & 3.40 & 1.58 & 7.30 \\
\hline Disorientation & 18 & 4 & 2.72 & 1.05 & 7.07 \\
\hline Cytokinereleasesyndrome & 498 & 137 & 2.47 & 2.22 & 2.74 \\
\hline Malaise & 24 & 6 & 2.42 & 1.17 & 5.02 \\
\hline Confusionalstate & 43 & 11 & 2.38 & 1.47 & 3.84 \\
\hline Neutropenia & 43 & 11 & 2.38 & 1.47 & 3.84 \\
\hline Tachycardia & 63 & 17 & 2.26 & 1.57 & 3.26 \\
\hline Fatigue & 38 & 14 & 1.64 & 1.04 & 2.61 \\
\hline Pyrexia & 187 & 90 & 1.27 & 1.08 & 1.50 \\
\hline \multicolumn{6}{|l|}{ FAERS } \\
\hline Events & $\mathrm{N}$ axicabtagene & $\mathrm{N}$ tisagenleucleucel & ROR & CI_low & CI_up \\
\hline Aphasia & 66 & 3 & 15.82 & 6.46 & 38.77 \\
\hline Car T-Cell-Related Encephalopathy Syndrome & 41 & 3 & 9.75 & 3.73 & 25.49 \\
\hline Atrial Fibrillation & 26 & 2 & 9.24 & 2.36 & 36.20 \\
\hline Thrombocytopenia & 36 & 5 & 5.13 & 2.49 & 10.53 \\
\hline Encephalopathy & 110 & 16 & 4.99 & 3.65 & 6.80 \\
\hline Disorientation & 28 & 4 & 4.97 & 2.10 & 11.77 \\
\hline Hyponatraemia & 14 & 2 & 4.96 & 1.10 & 22.33 \\
\hline Incontinence & 14 & 2 & 4.96 & 1.10 & 22.33 \\
\hline Tremor & 54 & 9 & 4.29 & 2.64 & 6.97 \\
\hline Transaminases Increased & 17 & 3 & 4.01 & 1.30 & 12.43 \\
\hline Neurotoxicity & 312 & 60 & 3.96 & 3.42 & 4.57 \\
\hline Somnolence & 41 & 8 & 3.65 & 2.10 & 6.34 \\
\hline Neutropenia & 57 & 17 & 2.39 & 1.64 & 3.48 \\
\hline Agitation & 20 & 6 & 2.36 & 1.10 & 5.08 \\
\hline Confusional State & 58 & 18 & 2.30 & 1.59 & 3.32 \\
\hline Mental Status Changes & 26 & 9 & 2.05 & 1.12 & 3.74 \\
\hline Disease Progression & 26 & 9 & 2.05 & 1.12 & 3.74 \\
\hline Cytokine Release Syndrome & 462 & 185 & 1.89 & 1.70 & 2.10 \\
\hline Tachycardia & 63 & 31 & 1.44 & 1.06 & 1.97 \\
\hline Pyrexia & 204 & 124 & 1.17 & 1.00 & 1.37 \\
\hline
\end{tabular}

$N$ number of reports, $R O R$ reporting odds ratio, $C I$ low lower bound of the $95 \%$ confidence interval, $C I \_$up upper bound of the $95 \%$ confidence interval

hepatic and musculoskeletal toxicities [22]. All these events have been reported for both drugs and in both databases. Different studies in literature account these events as the most common with CAR-T therapies [23-25]. In most cases, the trigger is the cytokine release syndrome that starts a subsequent cascade of events. The main goal is to prevent or at least limit the CRS to a low grade $[26,27]$. Some strategies in the management of CAR-T-related toxicities have been described such as pharmacological immunosuppression with tocilizumab or corticosteroids, suicide or elimination genes, and targeted activation [28, 29]. Recently, Lee at al. have described new methods that could prevent or rather terminate within $3 \mathrm{~h}$ the CRS-like toxicity by using low molecular weight adapters [30]. 
To date, IL-6 receptor antagonist tocilizumab represents the elective therapy in the management of the CRS. It was reported as concomitant drug in several reports of our survey, but it should be better considered as an emergency measure. As reported in its SPC, a minimum of four doses of tocilizumab should be available prior to infusion and during the monitoring period for use in the event of CRS. Neurotoxicity was the second most reported ADR, also referred to as CART-related encephalopathy syndrome. Several events reported in our analysis are symptoms of this toxicity such as encephalopathy, seizures, headache, aphasia, and memory impairment [31, 32]. A disproportionality has been highlighted for axicabtagene ciloleucel compared to tisagenlecleucel for both the abovementioned events: cytokine release syndrome and neurotoxicity. These data may suggest a higher association of these events to axicabtagene ciloleucel as compared to tisagenlecleucel. However, it should be remembered that the target population is different for the two CAR-T therapies, and this could influence the pattern and frequencies of the ADRs. Some rare and serious events have been reported such as multiple organ dysfunction syndrome, coagulopathies, included disseminated intravascular coagulation, and hemophagocytic lymphohistiocytosis. The complexity of the main event reported after the CAR-T treatment, i.e., the CRS, could also be at the root of these life-threatening ADRs [22, 33]. Inflammatory cells during CRS may lead to destruction of the integrity of endothelial barrier, thus causing CRS-related coagulopathy [34].

Among the most reported events, we found other hematological disorders: neutrophil count decreased, platelet count decreased, neutropenia, cytopenia, white blood cell count decreased, pancytopenia, and others. More than true adverse reactions to treatment, these are events related to the administration schedule, such as lymphodepleting chemotherapy with cyclophosphamide and fludarabine and leukapheresis before CAR-T infusions are the main culprits of these adverse reactions. As for the drugs reported as concomitant, some of them were drugs used in the administration procedure. For example, paracetamol and diphenhydramine are often used for pre-medication, allopurinol are used to reduce uric acid level in order to prevent tumor lysis syndrome in patient at risk, and corticosteroids and anti-seizure medicines such as levetiracetam are used for seizure prophylaxis. Some events related to the potential inefficacy of the treatment were also found, such as malignant neoplasm progression, acute lymphocytic leukemia recurrence, drug ineffectiveness (mainly for tisagenlecleucel), diffuse large B-cell lymphoma, acute lymphocytic leukemia, and B-cell-type acute leukemia. All these suggest the importance of continuing to evaluate data on approved drugs during their use in clinical practice. Our study also showed that the frequencies of the reported events were about the same for the two drugs in both databases. However, as shown in Table 1, they were lower than the frequencies highlighted in pre-marketing clinical trials. According to the safety information about axicabtagene
ciloleucel_-Yescarta ${ }^{\circledR}$ — in the ZUMA-1 study (108 patients enrolled), the most frequent ADRs were cytokine release syndrome (93\%), encephalopathy (58\%), and infections (39\%). Other ADRs reported as very common $(\geq 1 / 10)$ in the SPC are hematological disorders (leukopenia, neutropenia, anemia, and thrombocytopenia), tachycardia, arrhythmia, headache, tremor, and dizziness [6]. The safety assessment of tisagenlecleucel - Kymriah®_- was based on a total of 194 patients belonging to two clinical multicentre studies: CCTL019B2202 and CCTL019C2201. The most common ADRs were cytokine release syndrome $(77 \%$ in CCTL019B2202, 57\% in CCTL019C2201) and infections (73\% in CCTL019B2202, 58\% in CCTL019C2201); instead frequent hematological adverse reactions were decrease in lymphocytes (100\% in both the studies) and decrease in neutrophils (100\% in CCTL019B2202, 97\% in CCTL019C2201) [7].

These differences are probably due to the well-known limitation of ADR under-reporting in clinical practice $[35,36]$ that represents one of the limitations of our study. Furthermore, the same ADRs could have been reported with different terminology, and this may have led to distortion of their frequencies. In addition, the reports of suspected ADRs may be often incomplete and of poor quality, making difficult to obtain satisfactory data. This also made difficult the detection of duplicates. Furthermore, this type of study does not allow the quantification of the risk of an adverse event (in the absence of information about the number of exposed patients) but only gives a frequency description and suggests a possible association between drugs and adverse events. In any case, pharmacovigilance tools are used worldwide also by regulatory agencies to perform signal detection (i.e., the screening of safety information related to new ADR or to changed frequencies for already known ones) and are undoubtedly valuable, since they provide the highest volume of information at the lowest cost [37].

\section{Conclusion}

The type of adverse reactions reported from clinical practice is consistent with those in the SPCs of either drugs. The analysis of two of the largest databases worldwide such as EudraVigilance and FAERS has not revealed new signal, i.e., no new potential ADRs or increased frequencies. With the increasing use of these new therapies, a better definition of their real safety profile will be possible.

Author contribution Substantial contributions to conception or design of the work (G.B., D.M.), the acquisition (G.B., D.M.), analysis (G.B., N.M., A.V., D.M.), or interpretation of data for the work (G.B., N.M, A.V., D.M.). Drafting of the work (G.B., N. M, D.M.) or revising it critically for important intellectual content (N.M). All authors approved the submitted final version to be published. All authors agree to be 
accountable for all aspects of the work in ensuring that questions related to the accuracy or integrity of any part of the work are appropriately investigated and resolved.

Funding Open access funding provided by Alma Mater Studiorum Università di Bologna within the CRUI-CARE Agreement.

Data availability For this type of study, formal consent is not required.

\section{Declarations}

Conflict of interest The authors declare no competing interests.

Open Access This article is licensed under a Creative Commons Attribution 4.0 International License, which permits use, sharing, adaptation, distribution and reproduction in any medium or format, as long as you give appropriate credit to the original author(s) and the source, provide a link to the Creative Commons licence, and indicate if changes were made. The images or other third party material in this article are included in the article's Creative Commons licence, unless indicated otherwise in a credit line to the material. If material is not included in the article's Creative Commons licence and your intended use is not permitted by statutory regulation or exceeds the permitted use, you will need to obtain permission directly from the copyright holder. To view a copy of this licence, visit http://creativecommons.org/licenses/by/4.0/.

\section{References}

1. Neelapu SS, Locke FL, Bartlett NL, Lekakis LJ, Miklos DB, Jacobson CA, Braunschweig I, Oluwole OO, Siddiqi T, Lin Y, Timmerman JM, Stiff PJ, Friedberg JW, Flinn IW, Goy A, Hill BT, Smith MR, Deol A, Farooq U, McSweeney P, Munoz J, Avivi I, Castro JE, Westin JR, Chavez JC, Ghobadi A, Komanduri KV, Levy R, Jacobsen ED, Witzig TE, Reagan P, Bot A, Rossi J, Navale L, Jiang Y, Aycock J, Elias M, Chang D, Wiezorek J, Go WY (2017) Axicabtagene ciloleucel CAR T-cell in refractory large B-cell lymphoma. N Engl J Med 377:2531-2544

2. Maude SL, Laetsch TW, Buechner J, Rives S, Boyer M, Bittencourt H, Bader P, Verneris MR, Stefanski HE, Myers GD, Qayed M, de Moerloose B, Hiramatsu H, Schlis K, Davis KL, Martin PL, Nemecek ER, Yanik GA, Peters C, Baruchel A, Boissel N, Mechinaud F, Balduzzi A, Krueger J, June CH, Levine BL, Wood P, Taran T, Leung M, Mueller KT, Zhang Y, Sen K, Lebwohl D, Pulsipher MA, Grupp SA (2018) Tisagenlecleucel in children and young adults with B-cell lymphoblastic leukemia. N Engl J Med 378:439-448

3. Schuster SJ, Bishop MR, Tam CS, Waller EK, Borchmann P, McGuirk JP, Jäger U, Jaglowski S, Andreadis C, Westin JR, Fleury I, Bachanova V, Foley SR, Ho PJ, Mielke S, Magenau JM, Holte H, Pantano S, Pacaud LB, Awasthi R, Chu J, Anak Ö, Salles G, Maziarz RT (2019) Tisagenlecleucel in adult relapsed or refractory diffuse large B-cell lymphoma. N Engl J Med 380:45-56

4. Food and Drug Administration. Kymriah®. Available at: https:// www.fda.gov/vaccines-blood-biologics/cellular-gene-therapyproducts/kymriah-tisagenlecleucel. ()

5. Food and Drug Administration. Yescarta ${ }^{\circledR}$. Available at: https:// www.fda.gov/vaccines-blood-biologics/cellular-gene-therapyproducts/yescarta-axicabtagene-ciloleucel ()

6. European Medicine Agency. Yescarta ${ }^{\circledR}$ Summary of Product Characteristics Available at: https://www.ema.europa.eu/en/ documents/product-information/yescarta-epar-productinformation en.pdf. ()

7. European Medicine Agency. Kymriah ${ }^{\circledR}$ Summary of Product Characteristics Available at: https://www.ema.europa.eu/en/ documents/product-information/kymriah-epar-productinformation_en.pdf ()

8. Maude SL, Laetsch TW, Buechner J et al (2018) Tisagenlecleucel in children and young adults with B-cell lymphoblastic leukemia. N Engl J Med 378:439-448. https://doi.org/10.1056/ NEJMoa1709866

9. Schuster SJ, Bishop MR, Tam CS et al (2019) Tisagenlecleucel in adult relapsed or refractory diffuse large B-cell lymphoma. N Engl J Med 380:45-56. https://doi.org/10.1056/NEJMoa1804980

10. European Medicine Agency. European Public Assessment Report (EPAR) Kymriah. Available at: https://www.ema.europa.eu/en/ documents/assessment-report/kymriah-epar-public-assessmentreport_en.pdf ()

11. Locke FL, Ghobadi A, Jacobson CA et al (2019) Long-term safety and activity of axicabtagene ciloleucel in refractory large B-cell lymphoma (ZUMA-1): a single-arm, multicentre, phase 1-2 trial. Lancet Oncol 20:31-42. https://doi.org/10.1016/S1470-2045(18) 30864-7

12. European Medicine Agency. European Public Assessment Report (EPAR) Yescarta. Available at: https://www.ema.europa.eu/en/ documents/assessment-report/yescarta-epar-public-assessmentreport_en.pdf ()

13. Eudravigilance - European database of suspected adverse drug reaction reports. Available at: http://www.adrreports.eu/. ()

14. European Medicine Agency. New EudraVigilance System. Available at: https://www.ema.europa.eu/en/news/neweudravigilance-system-live ()

15. Brown EG, Wood L, Wood S (1999) The medical dictionary for regulatory activities (MedDRA). Drug Saf 20(2):109-117 PMID: 10082069

16. Salem JE, Manouchehri A, Moey M et al (2018) Cardiovascular toxicities associated with immune checkpoint inhibitors: an observational, retrospective, pharmacovigilance study. Lancet Oncol 19: 1579-1589. https://doi.org/10.1016/S1470-2045(18)30608-9

17. Zheng PP, Kros JM, Li J (2018 Jun) Approved CAR T cell therapies: ice bucket challenges on glaring safety risks and long-term impacts. Drug Discov Today 23(6):1175-1182. https://doi.org/10. 1016/j.drudis.2018.02.012

18. National Cancer Institute. Cancer Stat Facts: NHL - Diffuse Large B-Cell Lymphoma (DLBCL). Available at: https://seer.cancer.gov/ statfacts/html/dlbcl.html ()

19. National Cancer Institute. Cancer Stat Facts: Leukemia - Acute Lymphocytic Leukemia (ALL). Available at: https://seer.cancer. gov/statfacts/html/alyl.html ()

20. Inácio $\mathrm{P}$, Cavaco A, Airaksinen $\mathrm{M}(2017 \mathrm{Feb})$ The value of patient reporting to the pharmacovigilance system: a systematic review. $\mathrm{Br}$ J Clin Pharmacol 83(2):227-246. https://doi.org/10.1111/bcp. 13098

21. Giavridis T, Van der Stegen SJC, Eyquem J et al (2018) CAR T cell-induced cytokine release syndrome is mediated by macrophages and abated by IL-1 blockade. Nat Med 24(6):731-738. https://doi.org/10.1038/s41591-018-0041-7

22. Brudno JN, Kochenderfer JN (2016) Toxicities of chimeric antigen receptor T cells: recognition and management. Blood. 127(26): 3321-3330. https://doi.org/10.1182/blood-2016-04-703751

23. Smith L, Venella K (2017) Cytokine release syndrome: inpatient care for side effects of CAR T-cell therapy. Clin J Oncol Nurs 21(2):29-34

24. Li J, Wu Z, Zhao N (2019 Oct) Individual patient data metaanalysis from 16 trials for safety factors in cytokine release syndrome after CAR-T therapy in patients with non-Hodgkin 
lymphoma (NHL) and acute lymphoblastic leukemia. Adv Ther 36(10):2881-2894. https://doi.org/10.1007/s12325-019-01056-8

25. Frey N, Porter D (2019 Apr) Cytokine release syndrome with chimeric antigen receptor $\mathrm{T}$ cell therapy. Biol Blood Marrow Transplant 25(4):e123-e127. https://doi.org/10.1016/j.bbmt.2018. 12.756

26. Grigor EJM, Fergusson D, Kekre N (2019) Risks and benefits of chimeric antigen receptor T-cell (CAR-T) therapy in cancer: a systematic review and meta-analysis. Transfus Med Rev 33(2):98110. https://doi.org/10.1016/j.tmrv.2019.01.005

27. Porter D, Frey N, Wood PA, Weng Y, Grupp SA (2018) Grading of cytokine release syndrome associated with the CAR T cell therapy tisagenlecleucel. J Hematol Oncol 11(1):35. https://doi.org/10. 1186/s13045-018-0571-y

28. Bonifant CL, Jackson HJ, Brentjens RJ, Curran KJ (2016) Toxicity and management in CAR T-cell therapy. Mol Ther Oncolytics 3: 16011. https://doi.org/10.1038/mto.2016.11

29. Yu S, Yi M, Qin S, Wu K (2019) Next generation chimeric antigen receptor T cells: safety strategies to overcome toxicity. Mol Cancer 18:125. https://doi.org/10.1186/s12943-019-1057-4

30. Lee YG, Chu H, Lu Y et al (2019) Regulation of CAR T cellmediated cytokine release syndrome-like toxicity using low molecular weight adapters. Nat Commun 10(1):2681. https://doi.org/10. 1038/s41467-019-10565-7

31. Lee DW, Gardner R, Porter DL et al (2014) Current concepts in the diagnosis and management of cytokine release syndrome. Blood. 124(2):188-195. https://doi.org/10.1182/blood-2014-05-552729

32. Chavez JC, Jainb MD, Kharfan-Dabaja MA (2019) Cytokine release syndrome and neurologic toxicities associated with chimeric antigen receptor T-cell therapy: a comprehensive review of emerging grading models. Hematol Oncol Stem Cell Ther 13:1-6. pii: S1658-3876(19)30050-0. https://doi.org/10.1016/j.hemonc.2019. 05.005

33. Fitzgerald JC, Weiss SL, Maude SL et al (2017) Cytokine release syndrome after chimeric antigen receptor T cell therapy for acute lymphoblastic leukemia. Crit Care Med 45(2):e124-e131. https:// doi.org/10.1097/CCM.0000000000002053

34. Jiang H, Liu L, Guo T, Wu Y, Ai L, Deng J, Dong J, Mei H, Hu Y (2019) Improving the safety of CAR-T cell therapy by controlling CRS-related coagulopathy. Ann Hematol 98:1721-1732. https:// doi.org/10.1007/s00277-019-03685-z

35. Hazell L, Shakir SA (2006) Under-reporting of adverse drug reactions: a systematic review. Drug Saf 29(5):385-396. https://doi.org/ 10.2165/00002018-200629050-00003

36. Alvarez-Requejo A, Carvajal A, Beagaud B et al (1998) Underreporting of adverse drug reactions. Estimate based on a spontaneous reporting scheme and a sentinel system. Eur J Clin Pharmacol 54(6):483-488. https://doi.org/10.1007/s002280050498

37. Pal NS, Duncombe C, Falzon D, Olsson S (2013) WHO strategy for collecting safety data in public health programmes: complementing spontaneous reporting systems. Drug Saf 36(2):75-81. https://doi. org/10.1007/s40264-012-0014-6

Publisher's note Springer Nature remains neutral with regard to jurisdictional claims in published maps and institutional affiliations. 